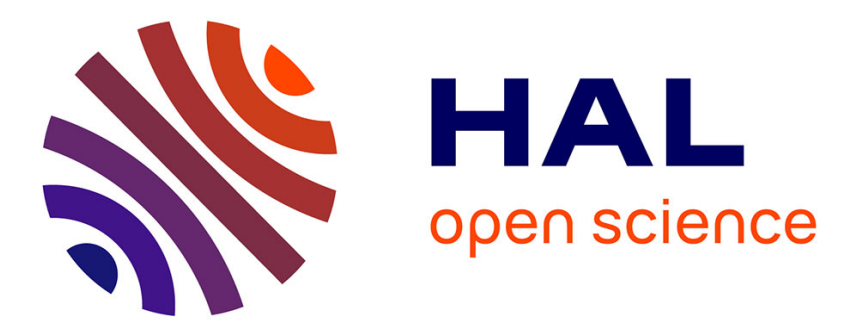

\title{
Towards control of plasma-induced surface roughness: simultaneous to plasma etching deposition
}

\author{
G. Kokkoris
}

\section{To cite this version:}

G. Kokkoris. Towards control of plasma-induced surface roughness: simultaneous to plasma etching deposition. European Physical Journal: Applied Physics, 2011, 56 (2), pp.24012. 10.1051/epjap/2011110205 . hal-00746207

\section{HAL Id: hal-00746207 \\ https://hal.science/hal-00746207}

Submitted on 28 Oct 2012

HAL is a multi-disciplinary open access archive for the deposit and dissemination of scientific research documents, whether they are published or not. The documents may come from teaching and research institutions in France or abroad, or from public or private research centers.
L'archive ouverte pluridisciplinaire HAL, est destinée au dépôt et à la diffusion de documents scientifiques de niveau recherche, publiés ou non, émanant des établissements d'enseignement et de recherche français ou étrangers, des laboratoires publics ou privés. 


\title{
Towards control of plasma induced surface roughness: Simultaneous to plasma etching deposition
}

George Kokkoris, Institute of Microelectronics, National Center for Scientific Research "Demokritos", Athens, Greece

Email: gkok@imel.demokritos.gr

Tel: +30 2106503267

Fax: +302106511723

\begin{abstract}
The potential of simultaneous to etching deposition for surface roughness control is investigated with a stochastic modeling framework for morphology evolution in $(2+1)$ d. It is predicted that ion-driven etching under simultaneous deposition of etchinhibitors (e.g. impurities coming from the wafer surroundings, the reactor walls, or the plasma bulk), not only induces roughness formation with a linear dependence on time, but can also induce periodic dots on the surface. The surface roughness can be controlled by regulating the amount of etch-inhibitors.

Title for running header: Control of surface roughness by simultaneous to plasma etching deposition
\end{abstract}

\section{Introduction}

The surface roughness induced by the fabrication processes in micro-/nanotechnology is crucial in a variety of fields. It can affect the antireflective [1] and the wetting properties of surfaces [2], the adsorption of proteins on surfaces [3], the cell growth [4], etc.. High roughness may be harmful or beneficial depending on the specific application. For example, a smooth hydrophobic surface becomes super-hydrophobic when its roughness increases.

Given the importance of surface roughness, its control becomes crucial. Since etching and deposition processes can produce surface roughness, they can be utilized to control the surface roughness. A reasonable argument is that the combination of etching and deposition would also induce surface roughness, and, by increasing the degrees of freedom (subtraction and/or addition of material), would make roughness control easier.

Even if etching and deposition processes were directed to be used separately, the combination of etching and deposition is inevitable in real etching/deposition systems and has been reported in the literature. The first example is plasma enhanced chemical vapor deposition (PECVD), where there is a small etching contribution due to the ions arriving on the surface. Unintentional co-deposition during plasma etching may be due to sputtered material from the - carrying the wafer - electrode [5] or from the reactor walls [6-8]. Co-deposition may also come from depositing species produced in the reactor bulk; this is the case in all etching recipes which are intended to build a passivating layer at the 
features' sidewall to achieve etching anisotropy [9, 10], including the gas chopping [11] or Bosch process and black Silicon (Si) method $[12,13]$. The so-called surface "grass" reported in the plasma etching literature was usually a result of co-deposition. Besides plasma etching processes, evidence of co-deposition was recently reported in ion beam sputtering (IBS) experiments and it was identified as a reason for surface roughness induction: Si surface roughness is induced by metal impurities coming from the ion source and sample surroundings [14-16]. The cause of roughness in all cases is the depositing species or etch-inhibitors which serve as local etching masks leading to etching rate non-uniformity.

Besides the unintentional co-deposition, there are experimental efforts [17, 18], initiated from the late $90 \mathrm{~s}$, where co-deposition is deliberate and intended to the formation of surface features on $\mathrm{Si}$ and other substrates. These efforts belong to the recently instituted and extremely active area of plasma aided nanofabrication [19, 20], which covers the lithography-less nano-pattern formation on surfaces by plasma processing. The majority of works for plasma aided nanofabrication use the "additive" or depositing mode of plasma processing [21] such as PECVD. Reported in the editorial review of a cluster issue on plasma aided fabrication of nano-structures and nanoassemblies [19], a milestone in the long term aim of achieving full control of nanoassembly is the understanding of the most effective "tuning-knobs" of plasma aided nano-fabrication. Given that the combination of two processes increases the degrees of freedom, the simultaneous to (plasma) etching deposition (SIMED) can be a crucial "tuning-knob" for the surface roughness control. The roughness control will be achieved through the control of deposition in terms of the type and the amount of depositing species.

The need of a model for SIMED, i.e. a model which includes the effect of impurities or etch-inhibitors or depositing particles for the explanation of pattern formation during IBS of Si was pinpointed by Macko et al. in a recent work [14]. There are interesting modeling works with focus on surface roughness and pattern formation which refer either to etching only [22-25] or deposition only [26, 27]. A first effort to model SIMED with a $(1+1)$ d stochastic modeling framework was reported in [28], where it was shown that SIMED could lead to an instability in roughness evolution versus time (linear increase of root mean square of roughness versus time) and dual scale surface roughness.

The present work is an extension of the previous one [28]. The modeling framework is extended to $(2+1) \mathrm{d}$ from $(1+1) \mathrm{d}$. The reason is that the key phenomenon of the roughness mechanisms proposed in [28], i.e. shadowing, is much more severe in $(1+1) d$ than in $(2+1)$ d. In addition, the results presented in this work also refer to a different value of etching selectivity of the etch inhibitors over the substrate. Furthermore, this work focuses also on the potential of SIMED to induce periodic dots on surfaces. In particular, it is shown that SIMED can induce periodic dots under an iondriven etching mechanism; this applies to both ion-enhanced plasma etching and IBS.

The importance of producing a periodic surface morphology stems from its usefulness in several fields such as in magnetic storage technology [29], catalysis [30], and nano-patterning where periodic surface morphology can be used as a mask for pattern transfer or as a mold. Usually, the formation of periodic morphology on surfaces can be achieved by two step processes including a lithographic step (e.g. nanoimprint, 
colloidal, block co-polymer lithography) [31, 32] which imposes the periodicity and then a plasma etching (pattern transfer) step. Favorably, the periodicity on a surface can be also achieved by one-step, lithography-less, processes such as ion beam sputtering [33, 34]. Recently, it was shown that periodic dots can be formed on plasma etched polymeric surfaces [35].

The paper is structured as follows: The modeling framework is described in section 2, the results and the discussion follow (section 3), and the conclusions are summarized in section 4.

\section{The modeling framework}

A stochastic modeling framework for surface morphology evolution in $(2+1) \mathrm{d}$ is exploited to apply roughening mechanisms. In the context of this framework, the etched film is represented by cubic cells (Fig. 1a) and the solid on solid approximation [36] is considered. Particles with user defined angular distributions impinge on the cellular morphology. No particle-particle collisions are considered due to the high Knudsen number of the flow in the valleys of the surface morphology; the mean free path is large due to the low pressure conditions during plasma etching compared to the dimensions of valleys. The trajectory of each particle is calculated until sticking on a cell. The interaction of particles with the cells is defined by a) the sticking probability and b) the etching yield. Monte Carlo method is used to sample stochastic variables from probability distributions defining the initial position and direction of the particle, the etching yield, the reemission probability, and the direction of reemitted particles.

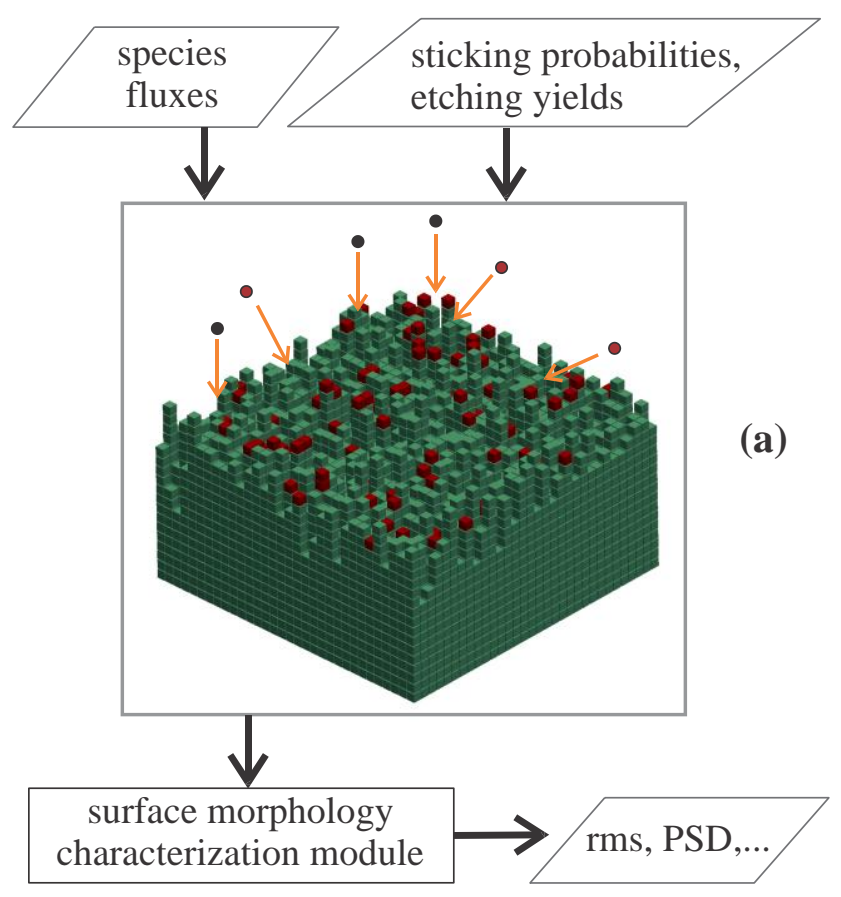




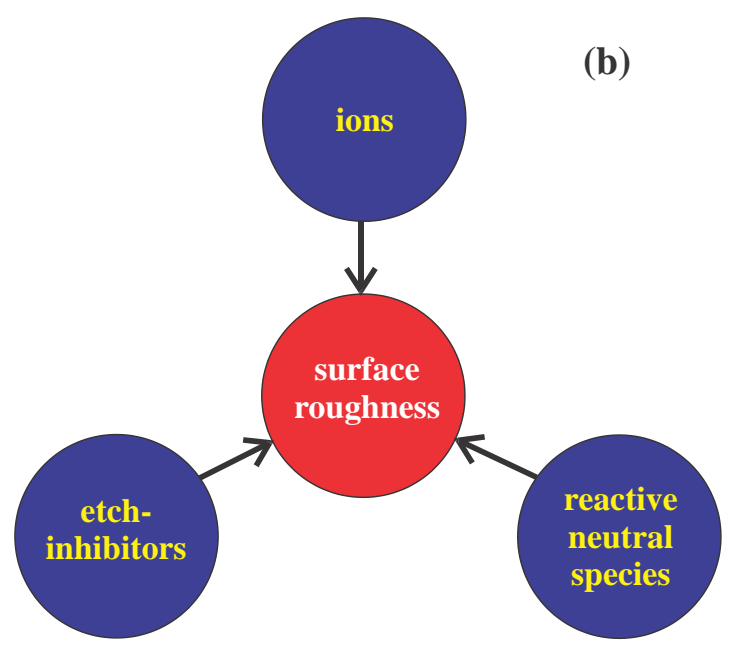

Figure 1. a) The flow chart of the modeling approach. b) Surface roughness of homogeneous substrates is the result of their interaction with ions, reactive neutral species, and etch-inhibitors.

The approach for the formulation of a mechanism for surface roughness formation is abstractive; the complex plasma environment is approximated with three generic-type species: Ions, depositing neutral species, which can be characterized as etch- inhibitors in an etching environment, and reactive neutral species, which cause etching of the substrate. The interaction of these species with the surface morphology will define its roughness (Fig. 1b).

In this work, the aim is to study the effect of SIMED on the surface morphology. In particular, the potential of simultaneous to ion-driven etching deposition for a) the roughness and b) periodic dots formation on surface morphologies is demonstrated. Two of the three types of species (see Fig. 1b) are employed: Ions and etch-inhibitors. This model is valid in cases where pure chemical etching is negligible and the ion-enhanced etching mechanism is dominant. Reactive neutral species are either in excess compared to ions and etch-inhibitors, so as to consider that the etched surface is fully covered by a thin layer of them, or absent. The latter case, where physical sputtering is the dominant mechanism, resembles with the case of IBS.

Regarding the conditions and inputs of the simulations presented in this work, the following set of assumptions are made. Ions reach the surface with a Gaussian angular distribution [37]; the standard deviation of the distribution is $2^{\circ}$. Their interaction with the surface is fundamental; no ion reflection or/and angle dependent sputtering are considered. The etch-inhibitors have an isotropic direction distribution, which is justified as they are neutral. They stick on the surface on first impact. Etching yields of both the substrate and the etch-inhibitors is 1 (a cell is removed per incident ion), i.e. etching selectivity of the substrate over the etch-inhibitors is 1 . The initial surface is always flat and its dimensions are $512 \times 512$ cells. The substrate is considered homogeneous.

Besides the modeling framework, critical for the evaluation of a roughness mechanism is a module for the surface characterization (see the flow chart in Fig. 1a). The surface roughness can be characterized by statistical properties of the surface morphology. Root mean square (rms) roughness or surface width is a common measure; it quantifies the vertical fluctuations of the surface morphology. The protrusions on a 
surface can be random or exhibit some periodicity. A measure to quantify the periodicity is the power spectral density (PSD). The emergence of a peak manifests periodicity. Further information on the statistical parameters of surface morphology as well as formulas for their calculations can be found in ref. [38].

\section{Results and discussion}

As noted in the introduction, the etch-inhibitors are coming from multiple sources in a plasma environment. Following an abstractive approach they can be categorized as [28] a) "hard" inhibitors, in the sense that they can be removed from the surface only by ions, and b) "soft" inhibitors, which can be removed from the surface by both ions and reactive neutral species. To separate the effect of each type of etch-inhibitors, two generic mechanisms for roughness formation and evolution were proposed [28], taking into account only "hard" (and ions) or only "soft" inhibitors (and reactive neutral species). In both mechanisms, random incidence of particles causes an initial roughness, i.e. small hills and valleys are formed (Figs. $2 \mathrm{a}$ and $2 \mathrm{~b}$ ). It is the preferential deposition of etchinhibitors on the hills compared to the valleys which causes the roughness increase. The origin of this preferential deposition on the hills is the more intense shadowing of etchinhibitors ("hard" or "soft") compared to ions (first mechanism) and to reactive neutral species (second mechanism). The difference in shadowing is due to different angular distributions of ions (almost normal to the surface) and "hard" inhibitors (isotropic) for the first mechanism, and due to the difference in the sticking probabilities of reactive neutral species and "soft" inhibitors for the second mechanism. Further details on the mechanisms can be found in ref. [28].

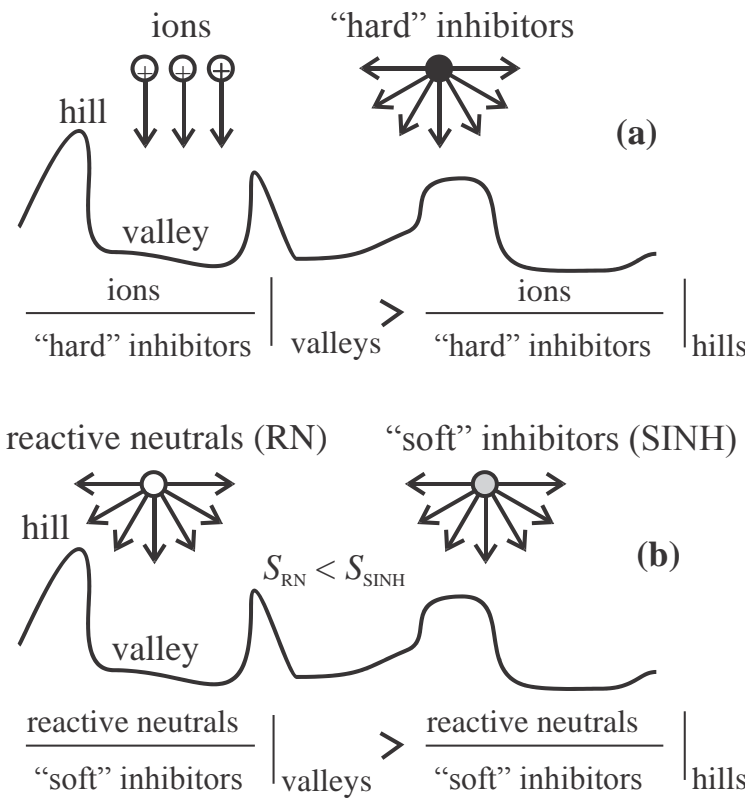

Figure 2. a) Mechanism for the roughness increase with "hard" inhibitors: The ratio of ions to inhibitors is higher in the valleys than in the hills, due to the difference in the angular distributions of inhibitors compared to the ions. b) Mechanism with "soft" 
inhibitors: The ratio of reactive neutral species to "soft" inhibitors is higher in the valleys than in the hills, due to the lower sticking probability of reactive neutral species compared to that of "soft" inhibitors $\left(S_{R N}<S_{S I N H}\right)$.

Evaluating the two mechanisms, it should be noticed that surface roughness can be induced by ions only as well; no etch-inhibitors are needed. The interesting point with the two mechanisms is that they can predict [28] the linear increase of rms roughness which a) is evident in experimental measurements of Si plasma etching [22, 28, 39-41], b) cannot be predicted when only ions cause the surface roughness, and c) is not an obvious outcome of micro-masking.

In Figs. 3 and 4, a comparison is performed between the case of ion-driven etching without "hard" inhibitors and the case where a small amount of them is present; the fraction of etch-inhibitors (ions) is $0.05(0.95)$ in the incident flux on the surface. The aim is to indicate the effect of etch-inhibitors on the surface morphology (Figs. 3a and 3b), rms roughness (Fig. 4a), and PSD (Fig. 4b).
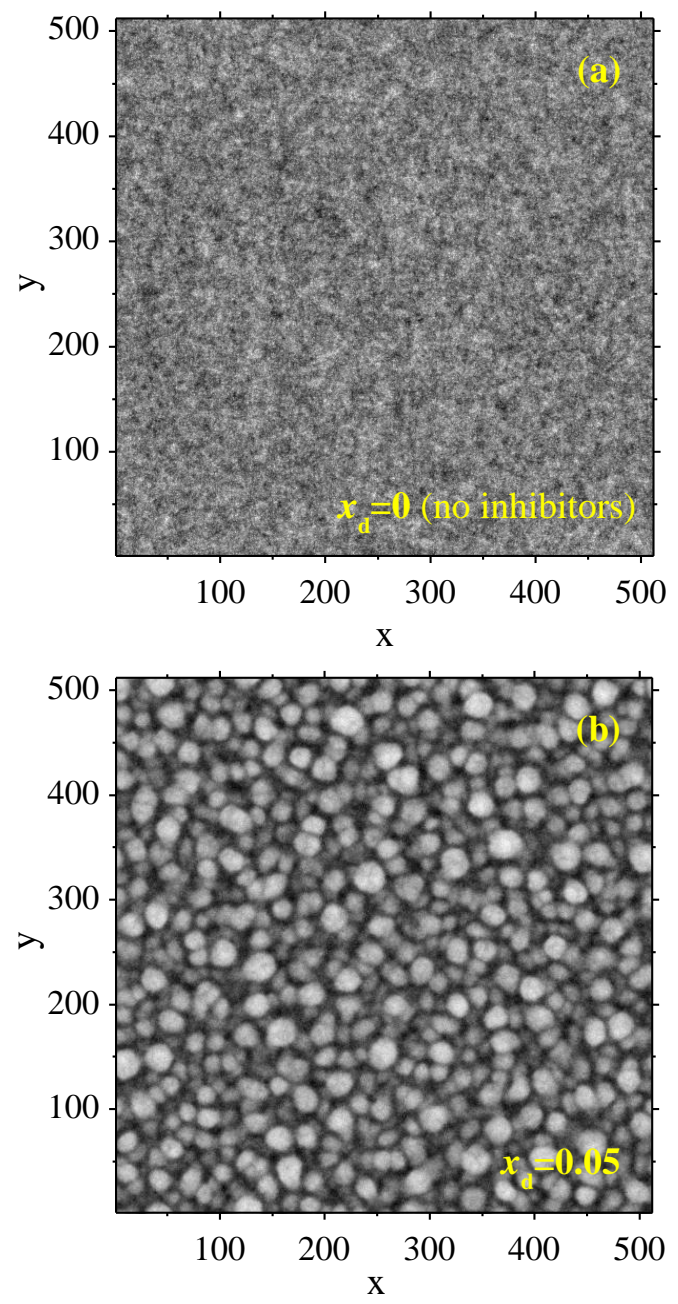

Figure 3. Top down surface morphologies under ion-driven etching a) without etchinhibitors and b) with a small amount of them in the incident flux; their fraction, $x_{\mathrm{d}}$, is 
0.05 . The morphologies are after $2400 \times N^{2}(N=512)$ incident particles. The conditions of the simulations are included in section 2 .

Comparing Figs. 3a and 3b, we observe dot formation only in the presence of etch-inhibitors. Roughness is developed in time for both cases as shown in Fig. 4a, following however a different power law, $t^{\beta}$, in each case. In particular, $\beta \approx 0.1$ in the absence of etch-inhibitors, and $\beta \approx 1$ when the etch-inhibitors are present.

It has to be noticed that $\beta \approx 1$ can be also produced by the mechanism proposed by Drotar et al. [22]. They considered a chemical etching mechanism and proposed a first order reemission mechanism to explain the rms roughness increase versus time. In particular, they considered that etching was caused by neutral species which were reemitted once. They assumed that the sticking probability was close to 0 for the first impact and 1 for the second. As a result, the valleys of the surface morphology received more etchant flux than the protrusions. Their model is really abstractive but their assumption for the difference of the sticking probability between the first and the second impact is not intuitive. Additionally, their first order reemission mechanism produces morphologies with holes [22, 23] and not with dots, as in the case of SIMED.

In Fig. 4b the circularly averaged PSD of surface morphologies under ion-driven etching with and without etch-inhibitors is shown. There is no peak in the PSD when etch-inhibitors are absent, while there is a peak when etch-inhibitors are present. The latter indicates that etch-inhibitors are necessary for the formation of periodic dots on the surface; only a small amount of them is enough.

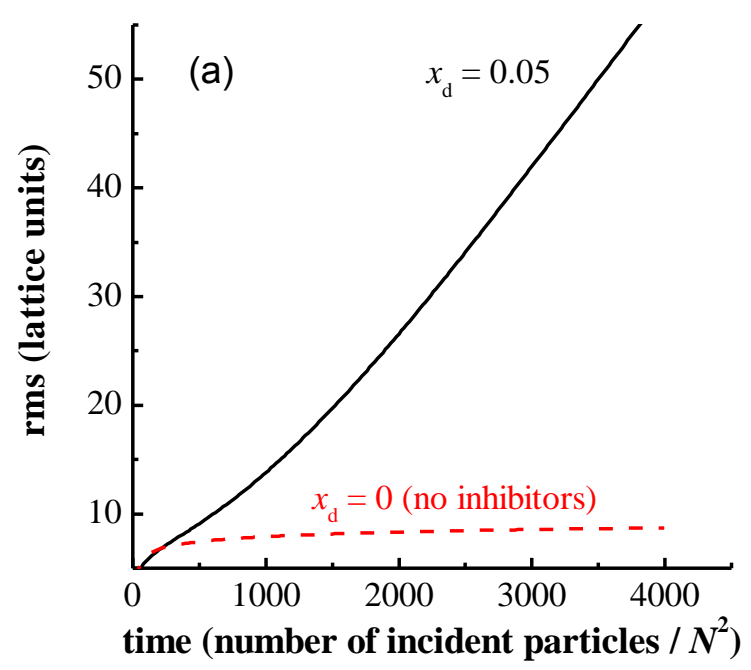




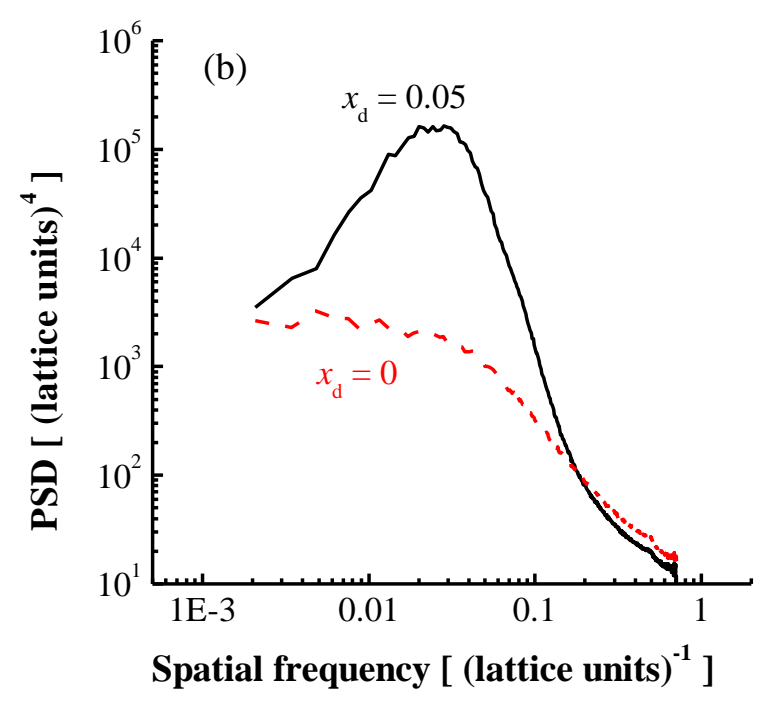

Figure 4. a) Rms roughness of surface morphology vs time (number of incident particles on the surface) under ion-driven etching without etch-inhibitors and with a small amount of them in the incident flux; their fraction, $x_{\mathrm{d}}$, is 0.05 . b) The PSD with and without etchinhibitors after $2400 \times N^{2}(N=512)$ incident particles. The rest of the simulation conditions are included in section 2 . The curves correspond to an average of 10 simulation runs.

It has to be noticed that the dots formed on the surface are not made by etchinhibitors. They consist predominantly of the substrate material. The amount of etchinhibitors on the surface is small, the surface coverage by etch-inhibitors is only 0.1 and it is almost constant during the process time. The surface coverage is defined as the ratio of the number of top cells which are etch-inhibitors divided by $N^{2}(N$ is the number of cells along $\mathrm{x}$ and $\mathrm{y}$ ). The surface coverage by etch-inhibitors increases as the amount of etch-inhibitors increases in the incident flux and causes an increase of the rms roughness of the surface morphology and a decrease of the etching yield (Fig. 5). Etching yield is defined as the ratio of the number of substrate cells removed by ions over the number of ions incident on the surface. It is equal to zero at a fraction equal to 0.5 ; after this value, deposition occurs on the surface. The results in Fig. 5 indicate that we can control the surface roughness (rms) of the surface morphologies by regulating the amount of etch inhibitors. The price to pay is a decrease of the etching yield, which is small at low fractions of etch-inhibitors: A 5\% decrease is calculated for a fraction equal to 0.05 . 


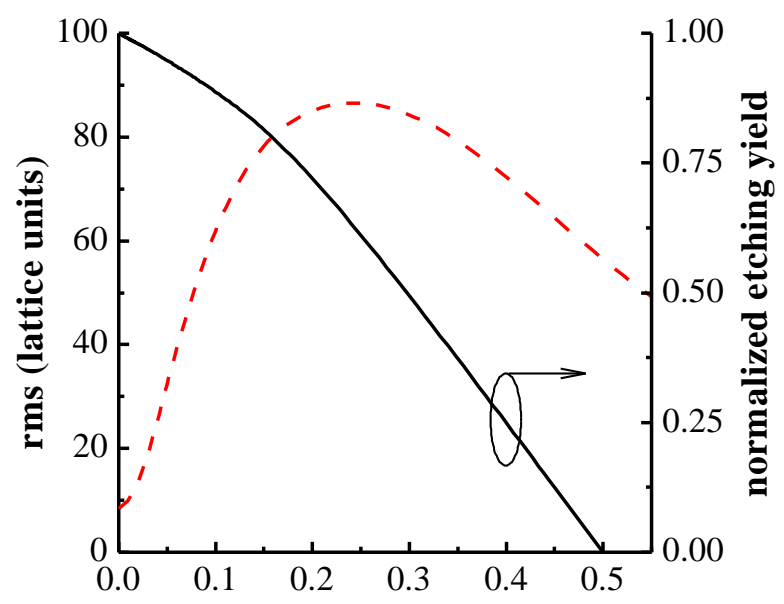

fraction of inhibitors in the incident flux

Figure 5. Rms roughness and normalized etching yield of surface morphology vs the fraction of the etch-inhibitors in the incident flux. The etching yield is normalized to its value in the case where etch-inhibitors are absent. The curves are the average of 10 simulation runs.

\section{Conclusions}

An abstractive, stochastic (Monte Carlo), $(2+1) \mathrm{d}$ modeling framework, used for the description of roughness formation and evolution on (plasma) etched surfaces, is utilized to show that SIMED or better the simultaneous to ion-driven etching deposition of etch-inhibitors can cause linear increase of rms versus time and periodic dots on etched surfaces of homogeneous substrates.

It has to be noticed that SIMED is not the only route to induce periodicity on surface morphologies and generally to control surface roughness. For example, the mechanisms of surface roughening in plasma treated polymeric surfaces may be attributed to stress related phenomena, the polymer structure, and ions - radiation synergy as pinpointed in a series of recent works [42-44]. However, SIMED has to be taken into account in cases where it is experimentally (e.g. by X-ray photoelectron spectroscopy) verified [7].

The theoretical experiments presented in this work verify the potential of SIMED for surface morphology control. SIMED seems a promising and challenging process towards the control of surface periodicity and generally surface roughness. On one hand, it is a process with more degrees of freedom (more variables to handle). On the other hand, it is a more complex process. A critical question arises for its practical implementation: Given the multiple sources of etch-inhibitors in a plasma reactor, how can we control their amount? 


\section{Acknowledgements}

The author would like to thank Dr. Vassilios Constantoudis for the discussion on the surface characterization, Dr. Evangelos Gogolides, and Dr. Angeliki Tserepi for the fruitful discussions on surface roughness induced by plasma etching.

\section{References}

1. S. Koynov, Brandt M.S., and Stutzmann M., Appl. Phys. Lett. 88, 203107 (2006)

2. D. Quere, Annual Review of Materials Research. 38, 71 (2008)

3. $\quad$ K. Tsougeni, et al., Langmuir. 26, 13883 (2010)

4. $\quad$ R. Gristina, et al., J. Biomed. Mater. Res. B. 88, 139 (2009)

5. G.S. Oehrlein, Schad R.G., and Jaso M.A., Surf. Interface Anal. 8, 243 (1986)

6. M. Martin and Cunge G., J. Vac. Sci. Technol. B. 26, 1281 (2008)

7. $\quad$ K. Tsougeni, et al., Langmuir. 25, 11748 (2009)

8. $\quad$ E. Gogolides, et al., J. Phys. D: Appl. Phys. 44, 174021 (2011)

9. G.S. Oehrlein, Rembetski J.F., and Payne E.H., J. Vac. Sci. Technol. B. 8, 1199 (1990)

10. R. Dussart, et al., J. Phys. D: Appl. Phys. 38, 3395 (2005)

11. I.W. Rangelow, J. Vac. Sci. Technol. A. 21, 1550 (2003)

12. H. Jansen, et al., J. Micromech. Microeng. 5, 115 (1995)

13. H.V. Jansen, et al., J. Micromech. Microeng. 20, 075027 (2010)

14. S. Macko, et al., Nanotechnology. 21, 085301 (2010)

15. J.A. Sanchez-Garcia, et al., Nanotechnology. 19, 355306 (2008)

16. J. Zhou, Hildebrandt M., and Lu M., J. Appl. Phys. 109, 053513 (2011)

17. K. Seeger and Palmer R.E., Appl. Phys. Lett. 74, 1627 (1999)

18. C.K. Park, et al., J. Vac. Sci. Technol. A. 25, 1073 (2007)

19. K. Ostrikov and Murphy A.B., J. Phys. D: Appl. Phys. 40, 2223 (2007)

20. K. Ostrikov, IEEE Trans. Plasma Sci. 35, 127 (2007)

21. J. Zheng, et al., Adv. Mater. 22, 1451 (2010)

22. J.T. Drotar, et al., Phys. Rev. B. 61, 3012 (2000)

23. Y.P. Zhao, et al., Phys. Rev. Lett. 82, 4882 (1999)

24. W. Guo, Bai B., and Sawin H.H., J. Vac. Sci. Technol. A. 27, 388 (2009)

25. W. Guo and Sawin H.H., J. Phys. D: Appl. Phys. 42, 194014 (2009)

26. M. Pelliccione, Karabacak T., and Lu T.M., Phys. Rev. Lett. 96, 146105 (2006)

27. T. Karabacak, Wang G.C., and Lu T.M., J. Appl. Phys. 94, 7723 (2003)

28. G. Kokkoris, et al., Phys. Rev. B. 76, 193405 (2007)

29. J. Shen and Kirschenr J., Surf. Sci. 500, 300 (2002)

30. G. Costantini, et al., J. Chem. Phys. 112, 6840 (2000)

31. A.K. Boal, et al., Nature. 404, 746 (2000)

32. Y.H. Ting, et al., J. Vac. Sci. Technol. B. 26, 1684 (2008)

33. F. Frost, et al., Appl. Phys. A. 91, 551 (2008)

34. J. Munoz-Garcia, et al. (Eds.), Self-organized surface nanopatterning by ion beam sputtering. (Springer, 2006)

35. N. Vourdas, et al., Nanotechnology. 21, 085302 (2010) 
36. A.L. Barabasi and Stanley H.E., Fractal Concepts in Surface Growth. (Cambridge University Press, 1995)

37. G. Kokkoris, Boudouvis A.G., and Gogolides E., J. Vac. Sci. Technol. A. 24, 2008 (2006)

38. Y. Zhao, Wang G.C., and Lu T.M., Experimental methods in the physical sciences. Vol. 37. Characterization of amorphous and crystalline rough surface: Principles and applications. (Academic Press, 2001)

39. G. Boulousis, et al., Nanotechnology. 19, 255301 (2008)

40. R. Petri, et al., J. Appl. Phys. 75, 7498 (1994)

41. P. Verdonck, et al., Journal of Solid-State Devices and Circuits. 6, 1 (1998)

42. $\quad$ R.L. Bruce, et al., J. Appl. Phys. 107, 084310 (2010)

43. D. Nest, et al., Plasma Processes and Polymers. 6, 649 (2009)

44. D. Nest, et al., J. Phys. D.: Appl. Phys. 43, 085204 (2010) 